\title{
A comparison principle for PDEs arising in approximate hedging problems: application to Bermudan options. ${ }^{* \dagger}$
}

\author{
Géraldine Bouveret \\ Department of Mathematics \\ Imperial College London \\ g.bouveret11@imperial.ac.uk
}

\author{
Jean-François Chassagneux \\ Laboratoire de Probabilités et Modèles Aléatoires \\ CNRS, UMR 7599, Université Paris Diderot \\ jean-francois.chassagneux@univ-paris-diderot.fr
}

September 11, 2018

\begin{abstract}
In a Markovian framework, we consider the problem of finding the minimal initial value of a controlled process allowing to reach a stochastic target with a given level of expected loss. This question arises typically in approximate hedging problems. The solution to this problem has been characterised by Bouchard, Elie and Touzi in [7] and is known to solve an Hamilton-Jacobi-Bellman PDE with discontinuous operator. In this paper, we prove a comparison theorem for the corresponding PDE by showing first that it can be rewritten using a continuous operator, in some cases. As an application, we then study the quantile hedging price of Bermudan options in the non-linear case, pursuing the study initiated in [3].
\end{abstract}

Keywords: stochastic target problems, comparison principle, quantile hedging, Bermudan options.

AMS 2010 Subject Classification: Primary, 49L25, 60J60, 93E20 ; Secondary, 49L20, $35 \mathrm{~K} 55$.

\section{Introduction}

On a filtered probability space, $\left(\Omega,\left(\mathcal{F}_{t}\right)_{t \geqslant 0}, \mathbb{P}\right)$, and given a terminal date $T>0$, we consider two diffusion processes $\left\{X_{s}^{t, x}, t \leqslant s \leqslant T\right\}$ and $\left\{Y_{s}^{t, x, y, \nu}, t \leqslant s \leqslant T\right\}$ with

${ }^{*}$ The final publication is available at Springer via http://dx.doi.org/[10.1007/s00245-017-9413-5].

${ }^{\dagger}$ This research has been sponsored by the Natixis Foundation for Quantitative Finance. 
values in respectively $(0, \infty)^{d}$ and $\mathbb{R}$ and initial conditions $(t, x) \in[0, T] \times(0, \infty)^{d}$ and $(t, x, y) \in[0, T] \times(0, \infty)^{d} \times \mathbb{R}$. The process $Y^{t, x, y, \nu}$ is controlled by $\nu$, a square integrable and progressively measurable process valued in $\mathbb{R}^{d}$. We are interested in the problem of finding the minimal initial value of a controlled process that allows to reach a target with a given level of expected loss, i.e.

$$
v(t, x, p):=\inf \left\{\begin{array}{c}
y \in \mathbb{R}^{+} \text {s.t. } \mathbb{E}\left[\ell \circ G\left(X_{T}^{t, x}, Y_{T}^{t, x, y, \nu}\right)\right] \geqslant p, \\
\text { for some admissible controls } \nu \in \mathbb{R}^{d}
\end{array}\right\},
$$

with $p \in I \subseteq \mathbb{R}, \ell$ a real-valued non-decreasing function and $G$ a real-valued function such that for $x \in(0, \infty)^{d}, y \mapsto G(x, y)$ is non-decreasing and $y \mapsto \ell \circ G(x, y)$ is rightcontinuous. Here, $I$ is an interval given by the closed convex hull of the image of $\ell \circ G$, namely $I:=\overline{\operatorname{conv}}\left(\ell \circ G\left((0, \infty)^{d} \times \mathbb{R}^{+}\right)\right)$.

Problem (1.1) is coined stochastic target problem with controlled loss by Bouchard, Elie and Touzi in [7] who considered a non-linear Markovian formulation in a Brownian diffusion setting. Moreau in [18] and Bouchard, Elie and Reveillac in [6] extended their results considering respectively jump diffusions and a non-Markovian setting. This problem arises when optimal management decisions are based on some risk criterion given by the loss function $\ell$. The latter belongs to the class of approximate hedging problems.

To obtain a PDE characterisation of $v$, Bouchard, Elie and Touzi in [7] first transformed the above problem into a stochastic target one in the $\mathbb{P}$-a.s. sense. To do so, they introduced an additional controlled state variable $P^{\alpha} \in I$ a.s. coming from the use of the martingale representation theorem. This reformulation allows then to use the geometric dynamic programming approach introduced by Soner and Touzi [20, 21] for a European constraint and by Bouchard and $\mathrm{Vu}$ in [9] for an obstacle constraint. However the additional controlled process in the increased state is unbounded leading to singular stochastic target problems and the Hamilton-Jacobi-Bellman equation, derived from the dynamic programming principle, involves a discontinuous operator.

The aim of this paper is to prove a comparison theorem for the PDE solved by $v$, opening the way to direct numerical methods to compute $v$. For example, one could build a convergent numerical scheme by adapting the generalised finite difference scheme defined by Bokanowski, Bruder, Maroso and Zidani in [1]. This method has to be compared to the dual algorithm proposed in [3], involving the computation of Fenchel transforms. We are indeed able to prove the comparison theorem under a setting involving a semi-linear dynamics for $Y$ and for unconstrained controls $\nu$. To the best of our knowledge, this is the first time that such a result is obtained in this non-linear setting. This answers a question raised in [5, Section 4] (preprint version of 
[6]). One key step is performed by using a renormalisation argument (see Section 3.1) to obtain a new continuous operator, in the spirit of [10]. However the new operator has a non-linearity in front of the time derivative. We therefore rely, as e.g. in [16], on a strict super-solution argument to prove that a comparison result holds (see Section 3.2 and Section 3.3).

To the best of our knowledge no comparison theorem has been proved so far for the PDE solved by $v$ in the case where the controls $\nu$ belong to a constrained set. This case corresponds to a constraint imposed on the gradients of the solution. This interesting problem is left for further research. Let us mention however, that using some approximation argument, Bouchard and $\mathrm{Vu}[8]$ are able to obtain a convergent numerical procedure to compute the value function $v$ at its continuity point, see also $[4]$.

In Section 4, we use our comparison result to characterise the quantile hedging price of Bermudan options in a non-linear setting, pursuing the study initiated in [3]. Quantile hedging problems have been introduced by Föllmer and Leukert (see [15]) for European-type claims and later studied by [7, 3, 17] amongst other. Precisely, we consider the problem of a trader who wants to find a hedging strategy $\nu$ and an initial endowment $y$ such that his hedging portfolio $Y^{t, x, y, \nu}$ stays above a claim of the form $g\left(\cdot, X^{t, x}\right)$ over a set of deterministic dates with a given probability. Here $X^{t, x}$ models the evolution of some risky assets, assuming that their value is $x$ at time $t$. The conservative case would be to ensure that the insurer meets a risk target over time almost surely. This is especially true in a Solvency II world where the risk should be monitored over time. However this constraint is too restrictive and we want to keep a flexible framework to allow for reasonable opportunities to make profits and this even with a limited available capital. Thus we weaken the constraint and express it in probability. In practice, the solvency constraint comes from an outside party, a minimal requirement for a fund manager, or the willingness to avoid a huge dis-utility. As usual in the Bermudan setting, the difficulty comes from the iteration over the time intervals of the characterisation obtained in the European case. In our case, the timeboundary condition on each interval is the most problematic issue since it involves a "facelift" phenomenon. Its full characterisation is obtained by using the continuity of the value function on the previous time interval, which comes from the application of the comparison theorem.

The rest of the paper is organised as follows. In Section 2, we introduce our framework for the study of stochastic target problems with controlled loss. In Section 3, we prove the comparison theorem. First, we obtain a new PDE characterisation for the 
value function involving a continuous operator. Then, we use a strict super-solution argument to obtain the comparison result. Finally, in Section 4, we study the quantile hedging price of a Bermudan option in our non-linear setting.

Notations. Let $d \geqslant 1$ be an integer. Any vector $x$ of $\mathbb{R}^{d}$ is seen as a column vector. We denote by $|x|$ the norm 1 of $x$, by $\|x\|$ its norm 2 and by $x^{\top}$ its transpose. The notation $\mathbb{M}^{d}$ denotes the set of $d$-dimensional square matrices and $\mathbb{S}^{d}$ is the subset of elements of $\mathbb{M}^{d}$ that are symmetric. We set $\mathrm{M}^{\top}$ the transpose of $\mathrm{M} \in \mathbb{M}^{d}$, while $\operatorname{Tr}[\mathrm{M}]$ is its trace. We respectively denote by $\mathbf{1}$ and $\mathbf{I}$ the $d$-dimensional unit column vector and the $d \times d$-dimensional unit matrix. Let $\psi:(t, x, p) \in[0, T] \times \mathbb{R}^{d} \times \mathbb{R} \mapsto \psi(t, x, p)$. If it is smooth enough, we denote by $\partial_{t} \psi$ its derivative with respect to $t$ and by $\mathrm{D} \psi$ its Jacobian matrix with respect to the space variables whose rows are given by $\mathrm{D}_{x} \psi$ and $\mathrm{D}_{p} \psi$, i.e. the derivative with respect to $x$ and $p$. The Hessian matrix with respect to the space variables is $\mathrm{D}^{2} \psi$ whose elements are given by $\mathrm{D}_{x x} \psi, \mathrm{D}_{p p} \psi, \mathrm{D}_{x p} \psi, \mathrm{D}_{p x} \psi$, i.e. the second derivative with respect to $x$ and $p$ and the cross derivatives. For a given function $f \in \mathbb{R}^{d}, f^{-1}$ stands for its inverse. Moreover, for a given function $f \in \mathbb{R}, \overline{\operatorname{conv}}(f)$ is the closed convex envelope of $f$.

Given a locally bounded map $v$ on an open subset $B$ of $\mathbb{R}^{d}$, we define the lower and upper semi-continuous envelopes

$$
v_{*}(b):=\liminf _{B \ni b^{\prime} \rightarrow b} v\left(b^{\prime}\right) \quad \text { and } \quad v^{*}(b):=\limsup _{B \ni b^{\prime} \rightarrow b} v\left(b^{\prime}\right), b \in \operatorname{cl}(B) .
$$

Finally $\mathcal{O}_{+}^{d}:=(0, \infty)^{d}$. All over the paper, inequalities between random variables have to be understood in the $\mathbb{P}$-a.s. sense.

\section{Problem statement}

In the sequel we work with a finite time horizon $T>0$. Let $\Omega$ be the space of $\mathbb{R}^{d}$-valued continuous functions $\left(\omega_{t}\right)_{t \leqslant T}$ on $[0, T], d \geqslant 1$, endowed with the Wiener measure $\mathbb{P}$. We denote by $W$ the coordinate mapping, i.e. $\left(W(\omega)_{t}\right)_{t \leqslant T}$ for $\omega \in \Omega$ so that $W$ is a $d$-dimensional Brownian motion on the canonical filtered probability space $(\Omega, \mathcal{F}, \mathbb{F}, \mathbb{P})$. In the latter $\mathcal{F}$ is the Borel tribe of $\Omega$ and $\mathbb{F}:=\left\{\mathcal{F}_{t}, 0 \leqslant t \leqslant T\right\}$ is the $\mathbb{P}$-augmentation of the filtration generated by $W$. Let $\mathcal{U}$ be the collection of $\mathbb{R}^{d}$-valued progressively measurable processes in $L^{2}([0, T] \times \Omega)$. For $t \in[0, T],(x, y) \in \mathcal{O}_{+}^{d} \times \mathbb{R}$ and $\nu \in \mathcal{U}$ the processes $X^{t, x}$ and $Y^{t, x, y, \nu}$ are defined as the solution to the following stochastic 
differential equations (SDE)

$$
\begin{aligned}
X_{s}^{t, x} & =x+\int_{t}^{s} \operatorname{diag}\left[X_{r}^{t, x}\right] \mu\left(X_{r}^{t, x}\right) \mathrm{d} r+\int_{t}^{s} \operatorname{diag}\left[X_{r}^{t, x}\right] \sigma\left(X_{r}^{t, x}\right) \mathrm{d} W_{r}, \\
Y_{s}^{t, x, y, \nu} & =y+\int_{t}^{s} \mu_{Y}\left(r, X_{r}^{t, x}, Y_{r}^{t, x, y, \nu}, \nu_{r}\right) \mathrm{d} r+\int_{t}^{s} \nu_{r}^{\top} \sigma\left(X_{r}^{t, x}\right) \mathrm{d} W_{r},
\end{aligned}
$$

where $\mu: \mathcal{O}_{+}^{d} \rightarrow \mathbb{R}^{d}, \sigma: \mathcal{O}_{+}^{d} \rightarrow \mathbb{M}^{d}$ and $\mu_{Y}:[0, T] \times \mathcal{O}_{+}^{d} \times \mathbb{R} \times \mathbb{R}^{d} \rightarrow \mathbb{R}$ are continuous functions.

We assume that $X^{t, x}$ takes its value in $\mathcal{O}_{+}^{d}$ when the initial condition $x$ is in $\mathcal{O}_{+}^{d}$. Moreover the process $Y^{t, x, y, \nu}$ is valued in $\mathbb{R}$. To guarantee that the above processes are well defined, we shall use throughout the paper the following standing assumption.

\section{Standing Assumption}

1. For all $\left(r, r^{\prime}, x, x^{\prime}, y, y^{\prime}, v, v^{\prime}\right) \in[0, T]^{2} \times\left(\mathcal{O}_{+}^{d}\right)^{2} \times \mathbb{R}^{2} \times \mathbb{R}^{2 d}$

$$
\begin{aligned}
& \left|\mu_{Y}\left(r^{\prime}, x^{\prime}, y^{\prime}, v^{\prime}\right)-\mu_{Y}(r, x, y, v)\right| \\
& \leqslant L\left(\left|r^{\prime}-r\right|+\left|x^{\prime}-x\right|\left(1+\left|v^{\prime}\right|+|v|\right)+\left|v^{\prime}-v\right|+\left|y^{\prime}-y\right|\right),
\end{aligned}
$$

for some Lipschitz constant $L>0$.

2. The functions $\mu_{X}: x \in \mathcal{O}_{+}^{d} \mapsto \operatorname{diag}[x] \mu(x) \in \mathbb{R}^{d}, \sigma_{X}: x \in \mathcal{O}_{+}^{d} \mapsto \operatorname{diag}[x] \sigma(x) \in \mathbb{S}^{d}$ are Lipschitz continuous with some Lipschitz constant $L>0$.

3. The function $\sigma$ is invertible and $x \in \mathcal{O}_{+}^{d} \mapsto \sigma^{-1}(x) \in \mathbb{S}^{d}$ is $L$-Lipschitz continuous on $\mathcal{O}_{+}^{d}$, for some $L>0$. The function $\mu, \sigma$ and $\sigma^{-1}$ are bounded by a constant $\Lambda>0$.

Finally, we shall sometimes use the following monotonicity assumption.

Assumption 2.0.1 (Drift monotonicity). The function $y \mapsto \mu_{Y}(\cdot, y, \cdot)$ is increasing.

Remark 2.1. It is well known that, in our context, setting $\tilde{Y}_{t}:=e^{\lambda t} Y_{t}, t \in[0, T]$, we obtain that the drift of $\tilde{Y}$ satisfies Assumption 2.0.1 when (2.1) is in force and provided that $\lambda$ is big enough.

To ease the notations, we will thus simply assume later in the proof of the comparison principle that Assumption 2.0.1 is in force. This is made clear in Remark 3.3 below.

Then we denote by $\mathcal{U}_{t, x, y}$ the subset of $\mathcal{U}$ for which the process $Y^{t, x, y, \nu} \geqslant 0$ on $[t, T]$.

Remark 2.2. In a financial setting the process $X^{t, x}$ is an underlying process representing the price of some risky assets while the process $Y^{t, x, y, \nu}$ is the wealth process where 
the control $\nu^{i}$ stands for the amount invested in assets $i$. In our setting two typical examples of $\mu_{Y}$ satisfy (2.1):

(i) The usual case of linear pricing, where there is a risk premium $\zeta(x):=\sigma^{-1}(x)(\mu(x)-$ $r \mathbf{1})$ with $r$ a risk-free interest rate and $\mu_{Y}(x, y, v):=\zeta^{\top}(x) \sigma(x) v$.

(ii) A case of non-linear pricing, coming from a market imperfection when there are two non-negative rates $R$ (the borrowing rate) and $r$ (the lending rate) with $R>r$ [14, Example 1.1]

$$
\mu_{Y}(x, y, v)=r y+\zeta(x)^{\top} \sigma(x) v-(R-r)\left(y-v^{\top} \mathbf{1}\right)_{-},
$$

where $\zeta$ stands for the risk premium.

Now let $\ell: \mathbb{R} \rightarrow \mathbb{R}$ be a non-decreasing function and $G: \mathbb{R}^{d+1} \mapsto \mathbb{R}$ be a measurable map such that for any $x \in \mathcal{O}_{+}^{d}, y \mapsto G(x, y)$ is non-decreasing and $y \mapsto \ell \circ G(x, y)$ is right-continuous. We also assume that $I:=\overline{\operatorname{conv}}\left(\ell \circ G\left(\mathcal{O}_{+}^{d} \times \mathbb{R}^{+}\right)\right)$, the closed convex hull of the image of $\ell \circ G$, is a compact interval of $\mathbb{R}$. In our application below, it is clear that - up to a proper rescaling of the $\ell$ function - one can consider that $I=[0,1]$ and we shall work under this setting from now on. For $(t, x, p) \in[0, T] \times \mathcal{O}_{+}^{d} \times[0,1]$, we then define the stochastic target problem with controlled loss as

$$
v(t, x, p):=\inf \left\{y \in \mathbb{R}^{+}: \exists \nu \in \mathcal{U}_{t, x, y} \text { s.t. } \mathbb{E}\left[\ell \circ G\left(X_{T}^{t, x}, Y_{T}^{t, x, y, \nu}\right)\right] \geqslant p\right\} .
$$

Assumption 2.0.2. We assume that there exists a constant $\beta>0$ such that

$$
|v(t, x, 1)| \leqslant \beta\left(1+|x|^{k}\right), k \geqslant 1, \text { for all }(t, x) \in[0, T] \times \mathcal{O}_{+}^{d} .
$$

Remark 2.3. Since, for all $p \in[0,1], 0 \leqslant v(\cdot, p) \leqslant v(\cdot, 1)$ the previous assumption implies the condition (2.3) holds true for $v$ on $[0, T] \times \mathcal{O}_{+}^{d} \times[0,1]$.

We assume that $\ell \circ G\left(X_{T}^{t, x}, Y_{T}^{t, x, y, \nu}\right)$ is square integrable for all initial conditions and for all $\nu \in \mathcal{U}_{t, x, y}$. Bouchard, Elie and Touzi proved in [7] that, in that case, (2.2) can be reduced to

$$
v(t, x, p)=\inf \left\{y \in \mathbb{R}^{+}: \exists(\nu, \alpha) \in \mathcal{U}_{t, x, y} \times \mathcal{A}_{t, p} \text { s.t. } \ell \circ G\left(X_{T}^{t, x}, Y_{T}^{t, x, y, \nu}\right) \geqslant P_{T}^{t, p, \alpha}\right\},
$$

where for $p \in[0,1], \mathcal{A}_{t, p}$ is the set of $\mathbb{R}^{d}$-valued $\mathbb{F}$-progressively measurable and square integrable processes $\alpha$ such that

$$
P_{T}^{t, p, \alpha}:=p+\int_{t}^{T} \alpha_{s}^{\top} \mathrm{d} W_{s} \in[0,1]
$$


As a consequence the problem reduction implies to work with an unbounded set of controls whatever the set of controls $\mathcal{U}$ is and then to deal with a discontinuous HamiltonJacobi-Bellman operator. The latter makes the proof of a comparison result difficult. The aim of this paper is thus to provide a comparison principle in the above framework (see Section 3). We will use this result to provide a full PDE characterisation of the quantile hedging price of a Bermudan option in our non-linear framework (see Section $4)$.

\section{A comparison principle}

In this section we prove a comparison principle for the PDE satisfied by the value function given in (2.2). As observed in [5, Section 4] (preprint version of [6]), this is not straightforward as this PDE is naturally obtained using a discontinuous operator, see equations (3.1)-(3.2) below. In a first step, we are able to show that any solution to this PDE can be characterised by a PDE involving a continuous operator. However this operator is non-standard as it involves a non-linearity in the time-derivative. Nevertheless, using a strict super-solution approach, we manage to prove a comparison theorem for this new PDE (and thus the original one).

\subsection{Alternative PDE characterisation inside the domain}

Let us start with some definitions. For $(t, x, y) \in[0, T] \times \mathcal{O}_{+}^{d} \times \mathbb{R}^{+}, q:=\left(\begin{array}{c}q^{x} \\ q^{p}\end{array}\right) \in \mathbb{R}^{d+1}$ and $A:=\left(\begin{array}{cc}A^{x x} & A^{x p} \\ A^{x p^{\top}} & A^{p p}\end{array}\right) \in \mathbb{S}^{d+1}$, denoting $\Xi:=(t, x, y, q, A)$, we define

$$
\mathrm{F}^{*}(\Xi):=\limsup _{\Xi^{\prime} \rightarrow \Xi} \mathrm{F}\left(\Xi^{\prime}\right)
$$

with

$$
\mathrm{F}(\Xi):=\sup _{(v, a) \in \mathcal{N}(x, y, q)}\left\{\mu_{Y}(t, x, y, v)-\mu_{X}^{\top}(x) q^{x}-\frac{1}{2} \operatorname{Tr}\left[\bar{\sigma} \bar{\sigma}^{\top}(x, a) A\right]\right\}
$$

where

$$
\mathcal{N}(x, y, q):=\left\{(v, a) \in \mathbb{R}^{d} \times \mathbb{R}^{d}: v^{\top} \sigma(x):=q^{\top} \bar{\sigma}(x, a)\right\} \text { and } \bar{\sigma}(x, a):=\left(\begin{array}{c}
\sigma_{X}(x) \\
a^{\top}
\end{array}\right),
$$

and where we recall the notations

$$
\mu_{X}(x):=\operatorname{diag}[x] \mu(x) \quad \sigma_{X}(x):=\operatorname{diag}[x] \sigma(x) .
$$


Let us observe that as $\sigma$ is invertible the previous expression can be simplified as $v$ is then a function of the variable $a$. We thus introduce

$$
J^{a}(\Xi)=\hat{\mu}_{Y}(t, x, y, q, a)-\mu_{X}^{\top}(x) q^{x}-\frac{1}{2} \operatorname{Tr}\left[\bar{\sigma} \bar{\sigma}^{\top}(x, a) A\right],
$$

where $\hat{\mu}_{Y}(t, x, y, q, a):=\mu_{Y}\left(t, x, y,\left(q^{\top} \bar{\sigma}(x, a) \sigma(x)^{-1}\right)^{\top}\right)$ and observe that

$$
\mathrm{F}(\Xi)=\sup _{a \in \mathbb{R}^{d}} J^{a}(\Xi)
$$

For the reader's convenience, we will write

$$
\mathrm{F} \varphi(t, x, p) \text { for } \mathrm{F}\left(t, x, \varphi(t, x, p), \partial_{t} \varphi(t, x, p), \mathrm{D} \varphi(t, x, p), \mathrm{D}^{2} \varphi(t, x, p)\right) .
$$

This writing will hold for any super-/sub-solution operator defined hereinafter.

Bouchard, Elie and Touzi in [7] proved that on $[0, T) \times \mathcal{O}_{+}^{d} \times(0,1), v^{*}$ is a viscosity sub-solution of

$$
\min \left\{v^{*},-\partial_{t} \varphi+\mathrm{F} \varphi\right\} \leqslant 0,
$$

and $v_{*}$ is a viscosity super-solution of

$$
-\partial_{t} \varphi+\mathrm{F}^{*} \varphi \geqslant 0
$$

As mentioned before the problem here stems from the fact that the Hamilton-JacobiBellman operator is lower semi-continuous and not upper semi-continuous.

As a consequence we will first work towards an alternative PDE characterisation of $v$ (see Theorem 3.1 below) that will allow us to express both the sub-solution and super-solution properties with a continuous operator.

Now let us denote by $\mathcal{S}$ the sphere of $\mathbb{R}^{d+1}$ of radius 1 and by $\mathcal{D}$ the set of vectors $\eta \in \mathcal{S}$ such that their first component $\eta^{1}=0$. For a vector $\eta \in \mathcal{S} \backslash \mathcal{D}$, we denote $\eta^{b}:=\frac{1}{\eta^{1}}\left(\eta^{2}, \ldots, \eta^{d+1}\right)^{\top} \in \mathbb{R}^{d}$. Moreover we define for $\Theta:=(t, x, y, b, q, A) \in[0, T] \times \mathcal{O}_{+}^{d} \times$ $\mathbb{R}^{+} \times \mathbb{R} \times \mathbb{R}^{d+1} \times \mathbb{S}^{d+1}$ the following operator

$$
\mathrm{H}^{\eta}(\Theta):=\left\{\begin{array}{cl}
\left(\eta^{1}\right)^{2}\left(-b+J^{\eta^{b}}(t, x, y, q, A)\right) & , \eta \in \mathcal{S} \backslash \mathcal{D} \\
-\frac{1}{2} A^{p p} & , \eta \in \mathcal{D}
\end{array} .\right.
$$

Observing that for $\eta \in \mathcal{S} \backslash \mathcal{D}$ the above operator reads

$\left(\eta^{1}\right)^{2}\left(-b+\hat{\mu}_{Y}\left(t, x, y, q, \eta^{b}\right)-\mu_{X}^{\top}(x) q^{x}-\frac{1}{2} \operatorname{Tr}\left[\sigma_{X} \sigma_{X}^{\top}(x) A^{x x}\right]-\frac{1}{2}\left\|\eta^{b}\right\|^{2} A^{p p}-\left(\eta^{b}\right)^{\top} \sigma_{X}^{\top}(x) A^{x p}\right)$, we can make the following remark. 
Remark 3.1. It follows from (2.1) that the operator $\eta \mapsto \mathrm{H}^{\eta}$ is continuous on $\mathcal{S}$, in particular,

$$
\sup _{\eta \in \mathcal{S}} \mathrm{H}^{\eta}(\Theta)=\sup _{\eta \in \mathcal{S} \backslash \mathcal{D}} \mathrm{H}^{\eta}(\Theta) .
$$

We can now state the alternative PDE characterisation of $v$.

Theorem 3.1. On $[0, T) \times \mathcal{O}_{+}^{d} \times(0,1), v^{*}\left(r e s p . v_{*}\right)$ is a viscosity sub-solution (resp. super-solution) of

$$
\mathcal{H} \varphi=0 \quad \text { with } \quad \mathcal{H}(\Theta)=\min \left\{y, \sup _{\eta \in \mathcal{S}} \mathrm{H}^{\eta}(\Theta)\right\},
$$

where $\Theta=(t, x, y, b, q, A) \in[0, T] \times \mathcal{O}_{+}^{d} \times \mathbb{R}^{+} \times \mathbb{R} \times \mathbb{R}^{d+1} \times \mathbb{S}^{d+1}$.

Proof. Step 1. Proof of the sub-solution property.

Let $\varphi$ be a smooth function such that $\max _{[0, T) \times \mathcal{O}_{+}^{d} \times(0,1)}\left(v^{*}-\varphi\right)(t, x, p)=\left(v^{*}-\varphi\right)\left(t_{0}, x_{0}, p_{0}\right)=$ 0 .

It follows from (3.1) that

$$
\min \left\{v^{*}\left(t_{0}, x_{0}, p_{0}\right),-\partial_{t} \varphi\left(t_{0}, x_{0}, p_{0}\right)+\mathrm{F} \varphi\left(t_{0}, x_{0}, p_{0}\right)\right\} \leqslant 0 .
$$

We will prove that

$$
-\partial_{t} \varphi\left(t_{0}, x_{0}, p_{0}\right)+\mathrm{F} \varphi\left(t_{0}, x_{0}, p_{0}\right) \leqslant 0 \Rightarrow \sup _{\eta \in \mathcal{S}} \mathrm{H}^{\eta} \varphi\left(t_{0}, x_{0}, p_{0}\right) \leqslant 0,
$$

which will lead to the sub-solution part of (3.3) as $v^{*} \geqslant 0$ by definition.

By definition of $\mathrm{F}$ we have that for all $a \in \mathbb{R}^{d}$,

$$
-\partial_{t} \varphi\left(t_{0}, x_{0}, p_{0}\right)+J^{a} \varphi\left(t_{0}, x_{0}, p_{0}\right) \leqslant 0 .
$$

For all $\eta \in \mathcal{S} \backslash \mathcal{D}$, we then obtain

$$
\left(\eta^{1}\right)^{2}\left(-\partial_{t} \varphi\left(t_{0}, x_{0}, p_{0}\right)+J^{\eta^{b}} \varphi\left(t_{0}, x_{0}, p_{0}\right)\right) \leqslant 0
$$

By continuity of $\eta \mapsto \mathrm{H}^{\eta}$, we thus obtain for all $\eta \in \mathcal{S}$,

$$
\mathrm{H}^{\eta} \varphi\left(t_{0}, x_{0}, p_{0}\right) \leqslant 0 .
$$

The arbitrariness of $\eta$ concludes the proof for this step.

Step 2. Proof of the super-solution property.

Let $\varphi$ be a smooth function such that $\min _{[0, T) \times \mathcal{O}_{+}^{d} \times(0,1)}\left(v_{*}-\varphi\right)(t, x, p)=\left(v_{*}-\varphi\right)\left(t_{0}, x_{0}, p_{0}\right)=$ 0 . We note that by definition $v_{*} \geqslant 0$ so that we just have to verify that

$$
\sup _{\eta \in \mathcal{S}} \mathrm{H}^{\eta} \varphi\left(t_{0}, x_{0}, p_{0}\right) \geqslant 0 .
$$


According to (3.2) we have,

$$
-\partial_{t} \varphi\left(t_{0}, x_{0}, p_{0}\right)+\mathrm{F}^{*} \varphi\left(t_{0}, x_{0}, p_{0}\right) \geqslant 0 .
$$

By definition of $\mathrm{F}^{*}$ we can find sequences $t_{k} \in[0, T),\left(x_{k}, p_{k}\right) \in \mathcal{O}_{+}^{d} \times(0,1), y_{k} \geqslant 0, q_{k}:=$ $\left(q_{k}^{x}, q_{k}^{p}\right) \in \mathbb{R}^{d+1}$, and a symmetric matrix $A_{k} \in \mathbb{S}^{d+1}$ such that

$$
\left(t_{k}, x_{k}, p_{k}\right) \rightarrow\left(t_{0}, x_{0}, p_{0}\right) \text { and }\left|\left(y_{k}, q_{k}, A_{k}\right)-\left(\varphi, \mathrm{D} \varphi, \mathrm{D}^{2} \varphi\right)\left(t_{0}, x_{0}, p_{0}\right)\right| \leqslant k^{-1},
$$

and

$$
-\partial_{t} \varphi\left(t_{0}, x_{0}, p_{0}\right)+\mathrm{F}\left(t_{k}, x_{k}, y_{k}, q_{k}, A_{k}\right) \geqslant-k^{-1} .
$$

Then we can find a maximising sequence $a_{k} \in \mathbb{R}^{d}$ such that

$$
-\partial_{t} \varphi\left(t_{0}, x_{0}, p_{0}\right)+J^{a_{k}}\left(t_{k}, x_{k}, y_{k}, q_{k}, A_{k}\right) \geqslant-2 k^{-1} .
$$

Now consider $\eta_{k}:=\left(\frac{1}{\sqrt{1+\left\|a_{k}\right\|^{2}}}, \frac{a_{k}}{\sqrt{1+\left\|a_{k}\right\|^{2}}}\right)^{\top}$. Note that $\eta_{k} \in \mathcal{S} \backslash \mathcal{D}$. Therefore we have

$$
\left(\eta_{k}^{1}\right)^{2}\left(-\partial_{t} \varphi\left(t_{0}, x_{0}, p_{0}\right)+J^{\eta_{k}^{b}}\left(t_{k}, x_{k}, y_{k}, q_{k}, A_{k}\right)\right) \geqslant-2 k^{-1}\left(\eta_{k}^{1}\right)^{2} .
$$

Hence using the relative compactness of the set $\mathcal{S} \backslash \mathcal{D}$ we have the existence of a subsequence such that $\lim _{k^{\prime} \rightarrow \infty} \eta_{k}^{\prime}=\bar{\eta}$ with $\bar{\eta} \in \mathcal{S}$. Moreover using (3.4), (2.1) and the standing hypotheses on the coefficients of $X$ we obtain

$$
\sup _{\eta \in \mathcal{S}} \mathrm{H}^{\eta} \varphi\left(t_{0}, x_{0}, p_{0}\right) \geqslant \mathrm{H}^{\bar{\eta}} \varphi\left(t_{0}, x_{0}, p_{0}\right) \geqslant 0,
$$

which concludes the proof for this step.

Remark 3.2. (1) We notice that the sub-solution property implies that $\mathrm{D}_{p p} \varphi\left(t_{0}, x_{0}, p_{0}\right) \geqslant$ 0 .

(2) Note that, if there exists a risk premium $\zeta(x):=\sigma^{-1}(x) \mu(x) \in \mathbb{R}^{d}$ (i.e. Remark 2.2

(i) with $r=0$ ) such that $\mu_{Y}(x, y, \nu):=\zeta^{\top}(x) \sigma(x) \nu$, (3.3) implies that $v^{*}$ is a viscosity sub-solution of

$$
\min \left\{v^{*}, \Lambda^{+}(\mathrm{M})\right\} \leqslant 0,
$$

and that $v_{*}$ is a viscosity super-solution of

$$
\Lambda^{+}(\mathrm{M}) \geqslant 0 \text {, }
$$

where $\Lambda^{+}(\mathrm{M})$ denotes the highest eigenvalue of the symmetric matrix $\mathrm{M}$ and where for $\Theta:=\left(x, b, q^{p}, A\right) \in \mathcal{O}_{+}^{d} \times \mathbb{R} \times \mathbb{R} \times \mathbb{S}^{d+1}$ the matrix $\mathrm{M}$ reads

$$
\mathrm{M}(\Theta):=\mathrm{M}_{1}(\Theta)+\mathrm{M}_{2}(\Theta)
$$


with $\mathrm{M}_{1}$ and $\mathrm{M}_{2}$ two $(d+1) \times(d+1)$ matrices defined as

$$
\mathrm{M}_{1}(\Theta):=\left(\begin{array}{cccc}
-b-\frac{1}{2} \operatorname{Tr}\left[\sigma_{X} \sigma_{X}^{\top}(x) A^{x x}\right] & 0 & \cdots & 0 \\
0 & -\frac{1}{2} A^{p p} & \cdots & 0 \\
\vdots & \vdots & \ddots & \vdots \\
0 & 0 & \cdots & -\frac{1}{2} A^{p p}
\end{array}\right) \text {, }
$$

and

$$
\mathrm{M}_{2}(\Theta):=\left(\begin{array}{cc}
0 & \frac{1}{2}\left[\zeta(x) q^{p}-\sigma_{X}(x) A^{x p}\right]^{\top} \\
\frac{1}{2}\left[\zeta(x) q^{p}-\sigma_{X}(x) A^{x p}\right] & 0
\end{array}\right) .
$$

This is in the spirit of [1, 2, 10].

Remark 3.3. By an usual change of variable argument, it is easily seen that if $v$ is a super-solution (resp. sub-solution) of

$$
\sup _{\eta \in \mathcal{S}} \mathrm{H}^{\eta} \varphi=0,
$$

then $\tilde{v}(t, x, p):=e^{\lambda t} v(t, x, p)$, for some $\lambda>0$, is a super-solution (resp. sub-solution) of

$$
\sup _{\eta \in \mathcal{S}} \tilde{\mathrm{H}}^{\eta} \varphi=0
$$

with

$$
\tilde{\mathrm{H}}^{\eta}(\Theta):=\left\{\begin{array}{cl}
\left(\eta_{1}\right)^{2}\left(-b+\tilde{J}^{\eta^{b}}(\Theta)\right) & , \eta \in \mathcal{S} \backslash \mathcal{D} \\
-\frac{1}{2} A^{p p} & , \eta \in \mathcal{D}
\end{array}\right.
$$

where

$$
\tilde{J}^{\eta^{b}}(\Theta)=\lambda y+e^{\lambda t} J^{\eta^{b}}\left(t, x, e^{-\lambda t} y, e^{-\lambda t} q, e^{-\lambda t} A\right) .
$$

For $\lambda>L$, we observe that $\tilde{J}^{\eta^{b}}$ is strictly increasing in $y$. From now on, we will thus assume that $\hat{\mu}_{Y}$ - and thus $J^{\eta^{b}}$ - is strictly increasing in y. Namely, we will assume that Assumption 2.0.1 is in force.

\subsection{Strict super-solution property and modulus of continuity}

The operator $\mathrm{H}^{\eta}$ has a non-linearity in front of the time-derivative. We then have to rely on a strict super-solution argument to prove that a comparison result holds for the non-linear PDE solved by $v$. This argument has been used, for example, by Ishii and Lions in [16] and Cheridito, Soner and Touzi in [11]. We thus have beforehand to introduce the following lemma. 
Lemma 3.1 (Strict super-solution property). Let us define on $[0, T] \times \mathcal{O}_{+}^{d} \times[0,1]$ the smooth positive functions $\phi(t, p):=e^{\kappa(T-t)}\left(\theta-\frac{e^{-4 \bar{c} p}}{2}\right), h(t, x):=e^{\kappa(T-t)}\left(|x|^{2 k}+|x|^{-2}\right)$, and

$$
f(t, x, p):=\phi(t, p)+h(t, x)>0,
$$

for some $\kappa, \theta>1$, with $\bar{c}:=L \times \Lambda$ (recall Standing Assumption) and $k$ introduced in Assumption 2.0.2.

Let $V$ be a lower semi-continuous super-solution of (3.3). Then, for $\kappa$ and $\theta$ big enough, the function $V+\xi f, \xi>0$, is a strict viscosity super-solution of (3.3) on $[0, T) \times \mathcal{O}_{+}^{d} \times(0,1)$, i.e. given a smooth function $\varphi$ such that $\min _{[0, T) \times \mathcal{O}_{+}^{d} \times(0,1)}((V+$ $\xi f)-\varphi)(t, x, p)=((V+\xi f)-\varphi)\left(t_{0}, x_{0}, p_{0}\right)=0$, one has

$$
\mathcal{H} \varphi\left(t_{0}, x_{0}, p_{0}\right) \geqslant \xi \varrho,
$$

for some $\varrho>0$.

Proof. Let $\varphi$ be a smooth function such that $\min _{[0, T) \times \mathcal{O}_{+}^{d} \times(0,1)}((V+\xi f)-\varphi)(t, x, p)=$ $((V+\xi f)-\varphi)\left(t_{0}, x_{0}, p_{0}\right)=0, \xi>0$. Since $f$ is a smooth function, the function $\psi:=\varphi-\xi f$ is a test function for $V$ at $\left(t_{0}, x_{0}, p_{0}\right)$.

We consider $\eta \in \mathcal{S} \backslash \mathcal{D}$, recall Remark 3.1. Using the definition of $\mathrm{H}^{\eta}$, the inequality

$$
\begin{aligned}
\hat{\mu}_{Y}(\cdot, & \left.(\psi+\xi f)\left(\cdot, p_{0}\right), \mathrm{D}(\psi+\xi f)\left(\cdot, p_{0}\right), \eta^{b}\right)\left(t_{0}, x_{0}\right) \\
\geqslant & \hat{\mu}_{Y}\left(\cdot, \psi\left(\cdot, p_{0}\right), \mathrm{D} \psi\left(\cdot, p_{0}\right), \eta^{\mathrm{b}}\right)\left(t_{0}, x_{0}\right) \\
& +\hat{\mu}_{Y}\left(\cdot, \psi\left(\cdot, p_{0}\right), \mathrm{D}(\psi+\xi f)\left(\cdot, p_{0}\right), \eta^{\mathrm{b}}\right)\left(t_{0}, x_{0}\right)-\hat{\mu}_{Y}\left(\cdot, \psi\left(\cdot, p_{0}\right), \mathrm{D} \psi\left(\cdot, p_{0}\right), \eta^{\mathrm{b}}\right)\left(t_{0}, x_{0}\right),
\end{aligned}
$$

(recall Remark 3.3) and the assumption on $\hat{\mu}_{Y}$, we obtain

$$
\mathrm{H}^{\eta} \varphi\left(t_{0}, x_{0}, p_{0}\right) \geqslant \mathrm{H}^{\eta} \psi\left(t_{0}, x_{0}, p_{0}\right)+\mathfrak{A}+\mathfrak{B},
$$

where

$$
\begin{aligned}
& \mathfrak{A}=\xi\left(\eta^{1}\right)^{2}\left(-\partial_{t} \phi-\bar{c}\left|\mathrm{D}_{p} \phi\right|\left|\eta^{b}\right|-\frac{1}{2}\left\|\eta^{b}\right\|^{2} \mathrm{D}_{p p} \phi\right)\left(t_{0}, x_{0}, p_{0}\right), \\
& \mathfrak{B}=\xi\left(\eta^{1}\right)^{2}\left(-\partial_{t} h-L\left|\operatorname{diag}[\cdot] \mathrm{D}_{x} h\right|-\left|\mu_{X}^{\top}(\cdot) \mathrm{D}_{x} h\right|-\frac{1}{2}\left|\operatorname{Tr}\left[\sigma_{X} \sigma_{X}^{\top}(\cdot) \mathrm{D}_{x x} h\right]\right|\right)\left(t_{0}, x_{0}, p_{0}\right) .
\end{aligned}
$$

We will now give a lower bound for both terms.

1. For the first term we compute, observing that $\partial_{t} \phi=-\kappa \phi, \mathrm{D}_{p} \phi(t, p)=2 \bar{c} e^{\kappa(T-t)-4 \bar{c} p}$ and $\mathrm{D}_{p p} \phi(t, p)=-8 \bar{c}^{2} e^{\kappa(T-t)-4 \bar{c} p}$,

$$
\begin{aligned}
\mathfrak{A} & =\xi\left(\eta^{1}\right)^{2} e^{\kappa\left(T-t_{0}\right)}\left(\kappa\left(\theta-\frac{e^{-4 \bar{c} p_{0}}}{2}\right)-2 \bar{c}^{2} e^{-4 \bar{c} p_{0}}\left|\eta^{b}\right|+4 \bar{c}^{2} e^{-4 \bar{c} p_{0}}\left\|\eta^{b}\right\|^{2}\right) \\
& \geqslant \xi\left(\eta^{1}\right)^{2} e^{\kappa\left(T-t_{0}\right)-4 \bar{c} p_{0}}\left((\theta-1)-2 \bar{c}^{2}\left(1+\left\|\eta^{\mathrm{b}}\right\|^{2}\right)+4 \bar{c}^{2}\left\|\eta^{b}\right\|^{2}\right),
\end{aligned}
$$


where we used the fact that $\kappa, \theta>1$.

Setting $\theta:=4 \bar{c}^{2}+1$, we obtain observing that $\left(\eta^{1}\right)^{2}\left(1+\left\|\eta^{\mathrm{b}}\right\|^{2}\right)=\|\eta\|^{2}=1$,

$$
\mathfrak{A} \geqslant 2 \xi \bar{c}^{2} e^{-4 \bar{c}} .
$$

2. For the second term, observing that

$$
\begin{gathered}
\partial_{t} h=-\kappa h, \quad \mathrm{D}_{x} h(t, x)=e^{\kappa\left(T-t_{0}\right)}\left(2 k|x|^{2 k-1}-2|x|^{-3}\right) \mathbf{1} \\
\text { and } \\
\mathrm{D}_{x x} h(t, x)=e^{\kappa\left(T-t_{0}\right)}\left(2 k(2 k-1)|x|^{2 k-2}+6|x|^{-4}\right) \mathbf{I},
\end{gathered}
$$

we compute,

$\mathfrak{B} \geqslant \xi\left(\eta^{1}\right)^{2} e^{\kappa\left(T-t_{0}\right)}\left(\kappa-2(k+1)(L+\Lambda)-\Lambda^{2} \frac{d}{2}(2 k(2 k-1)+6)\right)\left(|x|^{2 k}+|x|^{-2}\right) \geqslant \xi\left(\eta^{1}\right)^{2}$,

with $d$ the dimension of $X$ and for $\kappa$ large enough. In particular, we get $\mathfrak{B} \geqslant 0$.

Combining this last inequality with (3.6), we obtain

$$
\mathrm{H}^{\eta} \varphi\left(t_{0}, x_{0}, p_{0}\right) \geqslant \mathrm{H}^{\eta} \psi\left(t_{0}, x_{0}, p_{0}\right)+2 \xi \bar{c}^{2} e^{-4 \bar{c}} .
$$

3. We thus get that

$$
\sup _{\eta \in \mathcal{S} \backslash \mathcal{D}} \mathrm{H}^{\eta} \varphi\left(t_{0}, x_{0}, p_{0}\right) \geqslant \sup _{\eta \in \mathcal{S} \backslash \mathcal{D}} \mathrm{H}^{\eta} \psi\left(t_{0}, x_{0}, p_{0}\right)+2 \xi \bar{c}^{2} e^{-4 \bar{c}}
$$

We also observe that $V+\xi f \geqslant V+4 \xi \bar{c}^{2}$ with the above choice of $\theta$. The proof is concluded by using the super-solution property of $\psi$, recall Remark 3.1.

Lemma 3.2 (Modulus of continuity). Let $(b, x, r, p, q) \in \mathbb{R} \times\left(\mathcal{O}_{+}^{d}\right)^{2} \times[0,1]^{2}$ and $\left(y^{\prime}, y\right) \in$ $\mathbb{R}^{2}$ with $y^{\prime}>y$. Moreover, for $\varepsilon>0$, let $\mathcal{X}$ and $\mathcal{R} \in \mathbb{S}^{d+1} \times \mathbb{S}^{d+1}$ being such that

$$
\left(\begin{array}{cc}
\mathcal{X} & 0 \\
0 & -\mathcal{R}
\end{array}\right) \leqslant \frac{3}{\varepsilon}\left(\begin{array}{cc}
I & -I \\
-I & I
\end{array}\right)
$$

Setting $\delta=\frac{2}{\varepsilon}\left(\begin{array}{l}x-r \\ p-q\end{array}\right), \Theta=(t, r, y, b, \delta, \mathcal{R}), \Theta^{\prime}=\left(t, x, y^{\prime}, b, \delta, \mathcal{X}\right)$ then

$$
\sup _{\eta \in \mathcal{S}} \mathrm{H}^{\eta}(\Theta)-\sup _{\eta \in \mathcal{S}} \mathrm{H}^{\eta}\left(\Theta^{\prime}\right) \leqslant C\left(|x-r|+\frac{1}{\varepsilon}|x-r|^{2}(1+|x|+|r|)+\frac{1}{\varepsilon}|p-q|^{2}\right),
$$

for some constant $C>0$. 
Proof. Consider $\Theta$ and $\Theta^{\prime}$ defined in the theorem. We observe that

$$
\sup _{\eta \in \mathcal{S}} \mathrm{H}^{\eta}(\Theta)-\sup _{\eta \in \mathcal{S}} \mathrm{H}^{\eta}\left(\Theta^{\prime}\right) \leqslant \sup _{\eta \in \mathcal{S} \backslash \mathcal{D}}\left\{\mathrm{H}^{\eta}(\Theta)-\mathrm{H}^{\eta}\left(\Theta^{\prime}\right)\right\}
$$

(recall Remark 3.1).

For $\eta \in \mathcal{S} \backslash \mathcal{D}$, using the definition of $\mathrm{H}^{\eta}$ and the Lipschitz property of $\mu_{X}$, we then compute

$$
\mathrm{H}^{\eta}(\Theta)-\mathrm{H}^{\eta}\left(\Theta^{\prime}\right) \leqslant\left(\eta^{1}\right)^{2}\left(\mathfrak{A}+\frac{C}{\varepsilon}|x-r|^{2}+\mathfrak{B}\right),
$$

with $C>0$ and

$\mathfrak{A}=\hat{\mu}_{Y}\left(t, r, y, \delta, \eta^{b}\right)-\hat{\mu}_{Y}\left(t, x, y^{\prime}, \delta, \eta^{b}\right)$ and $\mathfrak{B}=-\frac{1}{2} \operatorname{Tr}\left[\bar{\sigma} \bar{\sigma}^{\top}\left(r, \eta^{b}\right) \mathcal{R}\right]+\frac{1}{2} \operatorname{Tr}\left[\bar{\sigma} \bar{\sigma}^{\top}\left(x, \eta^{b}\right) \mathcal{X}\right]$.

Since $y^{\prime}>y$, using the monotonicity property of $\hat{\mu}_{Y}$ (recall Remark 3.3) we have

$$
\begin{aligned}
\mathfrak{A} & \leqslant\left|\hat{\mu}_{Y}\left(t, r, y^{\prime}, \delta, \eta^{b}\right)-\hat{\mu}_{Y}\left(t, x, y^{\prime}, \delta, \eta^{b}\right)\right| \\
& \leqslant C\left(|x-r|+\frac{1}{\varepsilon}|x-r|^{2}(1+|x|+|r|)+\Lambda \frac{\left|\eta^{b}\right|}{\varepsilon}|x-r||p-q|+\frac{\left|\eta^{b}\right|}{\varepsilon}|p-q|\left|\sigma^{-1}(r)-\sigma^{-1}(x)\right|\right),
\end{aligned}
$$

where for the last inequality we used the definition of $\hat{\mu}_{Y}$, the Lipschitz property of $\mu_{Y}$ and the bound $\Lambda$ of $\sigma^{-1}$ (recall Standing Assumption). Using then Young's inequality and the Lipschitz continuity of $\sigma^{-1}$, we finally obtain

$$
\mathfrak{A} \leqslant C\left(1+\left|\eta^{b}\right|\right)\left(|x-r|+\frac{1}{\varepsilon}|x-r|^{2}(1+|x|+|r|)+\frac{1}{\varepsilon}|p-q|^{2}\right) .
$$

For the second order term $\mathfrak{B}$, we only have to use [12, Example 3.6], especially equation (3.7), recalling that $x \mapsto \bar{\sigma}(x, a)$ has the same Lipschitz constant as $\sigma_{X}$ by construction. We thus obtain

$$
\mathfrak{B} \leqslant \frac{C}{\varepsilon}|x-r|^{2}
$$

We thus have

$$
\mathrm{H}^{\eta}(\Theta)-\mathrm{H}^{\eta}\left(\Theta^{\prime}\right) \leqslant C\left(\eta^{1}\right)^{2}\left(1+\left|\eta^{b}\right|\right)\left(|x-r|+\frac{1}{\varepsilon}|x-r|^{2}(1+|x|+|r|)+\frac{1}{\varepsilon}|p-q|^{2}\right) .
$$

The proof is concluded by observing that $\left|\eta^{1}\right| \leqslant 1$ and $\left|\eta^{1} \eta^{\mathrm{b}}\right| \leqslant 1$. 


\subsection{The comparison principle}

We can now prove the comparison principle for (3.3).

Theorem 3.2. Let $V$ (resp. $U$ ) be a non-negative lower semi-continuous (resp. upper semi-continuous) map satisfying a polynomial growth of order $k \geqslant 1$ (defined in Assumption 2.0.2) on $[0, T] \times \mathcal{O}_{+}^{d} \times[0,1]$. Moreover assume that,

- on $[0, T) \times \mathcal{O}_{+}^{d} \times(0,1), U$ is a viscosity sub-solution of (3.3) and $V$ is a viscosity super-solution of (3.3),

- for all $(t, x) \in[0, T) \times \mathcal{O}_{+}^{d}, V(\cdot, 0) \geqslant U(\cdot, 0)$ and $V(\cdot, 1) \geqslant U(\cdot, 1)$,

- for all $(x, p) \in \mathcal{O}_{+}^{d} \times[0,1], V(T, \cdot) \geqslant U(T, \cdot)$,

then $V \geqslant U$ on $[0, T] \times \mathcal{O}_{+}^{d} \times[0,1]$.

Remark 3.4. The boundary conditions are necessary for the comparison to hold. Indeed let us assume that $\mu_{Y} \equiv 0$ and consider on $[0, T] \times[0,1]$ the function

$$
v_{\lambda, \gamma}(t, p):=2 T \kappa+(t-T)[\lambda p+\gamma(1-p)],(\lambda, \gamma) \in[0, \kappa] \times[0, \kappa],
$$

for some $\kappa>0$. Note that $v_{\lambda, \gamma}(T, \cdot)=2 T \kappa$ and $v_{\lambda, \gamma} \geqslant 0$ for all $(\lambda, \gamma) \geqslant[0, \kappa] \times[0, \kappa]$. We can easily prove that all the functions that belong to the family $\left(v_{\lambda, \gamma}(t, p)\right)_{\lambda, \gamma}$ are a viscosity sub-solution of (3.1) and a viscosity super-solution of (3.2). There is thus no unique solution to the system (3.1)-(3.2). However, if we set the following boundary conditions

$$
v_{\lambda, \gamma}(\cdot, 0)=2 T \kappa \quad \text { and } \quad v_{\lambda, \gamma}(\cdot, 1)=2 T \kappa,
$$

we thus obtain that $\lambda=\gamma=0$ and that $v_{0,0}=2 T \kappa$ is the unique solution to the above system.

Corollary 3.1. Assume that $v^{*}(\cdot, 0) \leqslant v_{*}(\cdot, 0)$ and $v^{*}(\cdot, 1) \leqslant v_{*}(\cdot, 1)$ on $[0, T) \times \mathcal{O}_{+}^{d}$ and assume that $v^{*}(T, \cdot) \leqslant v_{*}(T, \cdot)$ on $\mathcal{O}_{+}^{d} \times[0,1]$. Then the function $v$ is continuous on $[0, T] \times \mathcal{O}_{+}^{d} \times[0,1]$ and is the unique viscosity solution, in the class of function with polynomial growth, of

$$
\mathcal{H} v=0 \text { on }[0, T) \times \mathcal{O}_{+}^{d} \times(0,1) .
$$

Proof of Theorem 3.2 Let us now define on $(0, T] \times \mathcal{O}_{+}^{d} \times[0,1]$ the following nonnegative auxiliary function

$$
V_{\xi}(t, x, p):=(V+\xi f)(t, x, p)+\frac{\xi}{t},
$$

with $f$ defined in Lemma 3.1. Using Lemma 3.1, it is easily seen that $V_{\xi}$ is a strict super-solution of (3.3), namely it satisfies (3.5). 
We also introduce $U_{\xi}(t, x, p)=U(t, x, p)-\xi h(t, x)$, recall Lemma 3.1. By an easy adaptation of the proof of Lemma 3.1, we have that $U_{\xi}$ is still a sub-solution to (3.1).

We will show that $U-V \leqslant 0$ on $(0, T] \times \mathcal{O}_{+}^{d} \times[0,1]$. To this aim we will first prove by contradiction that for all $\xi>0$ we have $U_{\xi}-V_{\xi} \leqslant 0$ and the result will follow sending $\xi$ to zero.

Step 1. We assume to the contrary that there exists $\xi>0$ such that

$$
\sup _{(0, T] \times \mathcal{O}_{+}^{d} \times[0,1]}\left(U_{\xi}-V_{\xi}\right)(t, x, p)=\left(U_{\xi}-V_{\xi}\right)(\hat{t}, \hat{x}, \hat{p})=\gamma>0 .
$$

Observe that as $V_{\xi}>0$ the previous expression implies that

$$
U_{\xi}(\hat{t}, \hat{x}, \hat{p})>0 \text {. }
$$

For $\varepsilon>0$, we define on $(0, T] \times\left(\mathcal{O}_{+}^{d}\right)^{2} \times[0,1]^{2}$

$$
\Psi_{\varepsilon}(t, x, r, p, q):=U_{\xi}(t, x, p)-V_{\xi}(t, r, q)-\frac{1}{\varepsilon}\left(|x-r|^{2}+|p-q|^{2}\right) .
$$

Using the growth conditions and semi-continuity of $U$ and $V$, it follows that for $\varepsilon>0$ the function $\Psi_{\varepsilon}$ admits a maximum $M_{\varepsilon}$ at $\left(t_{\varepsilon}, x_{\varepsilon}, r_{\varepsilon}, p_{\varepsilon}, q_{\varepsilon}\right)$ on $(0, T] \times \mathcal{O}_{+}^{d^{2}} \times[0,1]^{2}$. Moreover the inequality $\Psi_{\varepsilon}\left(t_{\varepsilon}, x_{\varepsilon}, r_{\varepsilon}, p_{\varepsilon}, q_{\varepsilon}\right) \geqslant \Psi_{\varepsilon}(\hat{t}, \hat{x}, \hat{x}, \hat{p}, \hat{p})=\gamma$ combined with the growth condition on $U, V$ and the definition of $f$ and $h$, implies that $t_{\varepsilon}, x_{\varepsilon}$ and $r_{\varepsilon}$ are in compact set $\mathcal{T} \times \mathcal{K} \subset(0, T] \times \mathcal{O}_{+}^{d}$. Let $(\hat{t}, \hat{x}, \hat{p}) \in \mathcal{T} \times \mathcal{K} \times[0,1]$ be a limit point of $\left(t_{\varepsilon}, x_{\varepsilon}, p_{\varepsilon}\right)$. Using [12, Lemma 3.1] we obtain that

$$
\left\{\begin{array}{l}
\lim _{\varepsilon \downarrow 0} \frac{1}{\varepsilon}\left(\left|x_{\varepsilon}-r_{\varepsilon}\right|^{2}+\left|p_{\varepsilon}-q_{\varepsilon}\right|^{2}\right)=0, \\
\lim _{\varepsilon \downarrow 0} M_{\varepsilon}=\left(U_{\xi}-V_{\xi}\right)(\hat{t}, \hat{x}, \hat{p}) .
\end{array}\right.
$$

If $(\hat{t}, \hat{x}, \hat{p}) \in(0, T) \times \mathcal{O}_{+}^{d} \times\{0,1\}$ or $(\hat{t}, \hat{x}, \hat{p}) \in\{T\} \times \mathcal{O}_{+}^{d} \times[0,1]$, the assumptions on $V$ and $U$ on these boundaries of the domain lead to a contradiction.

We thus now assume that $0<\hat{t}<T$ and $0<\hat{p}<1$. In particular, up to a subsequence,

$$
U_{\xi}\left(t_{\varepsilon}, x_{\varepsilon}, p_{\varepsilon}\right)>0
$$

Step 2. From Ishii's Lemma (see [12, Theorem 8.3]) we get the existence of real coefficients $b_{\varepsilon}^{1}, b_{\varepsilon}^{2}$, a vector $d_{\varepsilon}$ and two symmetric matrices $\mathcal{X}_{\varepsilon}$ and $\mathcal{R}_{\varepsilon}$ being such that

$$
\left(b_{\varepsilon}^{1}, d_{\varepsilon}, \mathcal{X}_{\varepsilon}\right) \in \overline{\mathcal{J}}_{\overline{\mathcal{O}}}^{+} U_{\xi}\left(t_{\varepsilon}, x_{\varepsilon}, p_{\varepsilon}\right) \quad \text { and } \quad\left(-b_{\varepsilon}^{2}, d_{\varepsilon}, \mathcal{R}_{\varepsilon}\right) \in \overline{\mathcal{J}}_{\overline{\mathcal{O}}}^{-} V_{\xi}\left(t_{\varepsilon}, r_{\varepsilon}, q_{\varepsilon}\right),
$$


with $\overline{\mathcal{O}}:=(0, T) \times \mathcal{O}_{+}^{d} \times(0,1)$ and $\overline{\mathcal{J}}^{+}$(resp. $\left.\overline{\mathcal{J}}^{-}\right)$the limiting second order super-jet (resp. sub-jet) of $U_{\xi}\left(\right.$ resp. $\left.V_{\xi}\right)$ at $\left(t_{\varepsilon}, x_{\varepsilon}, p_{\varepsilon}\right) \in \overline{\mathcal{O}}\left(\right.$ resp. $\left.\left(t_{\varepsilon}, r_{\varepsilon}, q_{\varepsilon}\right) \in \overline{\mathcal{O}}\right)$ and where

$$
\begin{aligned}
b_{\varepsilon}^{1}+b_{\varepsilon}^{2} & :=0, \\
d_{\varepsilon} & :=\frac{2}{\varepsilon}\left(\begin{array}{c}
x_{\varepsilon}-r_{\varepsilon} \\
p_{\varepsilon}-q_{\varepsilon}
\end{array}\right), \\
\left(\begin{array}{cc}
\mathcal{X}_{\varepsilon} & 0 \\
0 & -\mathcal{R}_{\varepsilon}
\end{array}\right) & \leqslant \frac{3}{\epsilon}\left(\begin{array}{cc}
I & -I \\
-I & I
\end{array}\right) .
\end{aligned}
$$

It follows from the definition of $U_{\xi}$ and $V_{\xi}$ that they are respectively sub-/super-solution of (3.3). As a consequence using (3.9) and Lemma 3.1 we obtain

$$
\begin{aligned}
& \sup _{\eta \in \mathcal{S}} \mathrm{H}^{\eta}\left(t_{\varepsilon}, x_{\varepsilon}, U_{\xi}\left(t_{\varepsilon}, x_{\varepsilon}, p_{\varepsilon}\right), b_{\varepsilon}^{1}, d_{\varepsilon}, \mathcal{X}_{\varepsilon}\right) \leqslant 0, \\
& \sup _{\eta \in \mathcal{S}} \mathrm{H}^{\eta}\left(t_{\varepsilon}, r_{\varepsilon}, V_{\xi}\left(t_{\varepsilon}, r_{\varepsilon}, q_{\varepsilon}\right),-b_{\varepsilon}^{2}, d_{\varepsilon}, \mathcal{R}_{\varepsilon}\right) \geqslant \xi \rho>0 .
\end{aligned}
$$

Hence

$$
\sup _{\eta \in \mathcal{S}} \mathrm{H}^{\eta}\left(t_{\varepsilon}, r_{\varepsilon}, V_{\xi}\left(t_{\varepsilon}, r_{\varepsilon}, q_{\varepsilon}\right),-b_{\varepsilon}^{2}, d_{\varepsilon}, \mathcal{R}_{\varepsilon}\right)-\sup _{\eta \in \mathcal{S}} \mathrm{H}^{\eta}\left(t_{\varepsilon}, x_{\varepsilon}, U_{\xi}\left(t_{\varepsilon}, x_{\varepsilon}, p_{\varepsilon}\right), b_{\varepsilon}^{1}, d_{\varepsilon}, \mathcal{X}_{\varepsilon}\right) \geqslant \xi \rho .
$$

Step 3. On the other hand we know from Lemma 3.2 and (3.10) that there exists $C>0$ such that

$$
\begin{gathered}
\sup _{\eta \in \mathcal{S}} \mathrm{H}^{\eta}\left(t_{\varepsilon}, r_{\varepsilon}, V_{\xi}\left(t_{\varepsilon}, r_{\varepsilon}, q_{\varepsilon}\right),-b_{\varepsilon}^{2}, d_{\varepsilon}, \mathcal{R}_{\varepsilon}\right)-\sup _{\eta \in \mathcal{S}} \mathrm{H}^{\eta}\left(t_{\varepsilon}, x_{\varepsilon}, U_{\xi}\left(t_{\varepsilon}, x_{\varepsilon}, p_{\varepsilon}\right), b_{\varepsilon}^{1}, d_{\varepsilon}, \mathcal{X}_{\varepsilon}\right) \\
\leqslant C\left(\left|x_{\varepsilon}-r_{\varepsilon}\right|+\frac{1}{\varepsilon}\left|x_{\varepsilon}-r_{\varepsilon}\right|^{2}\left(1+\left|x_{\varepsilon}\right|+\left|r_{\varepsilon}\right|\right)+\frac{1}{\varepsilon}\left|p_{\varepsilon}-q_{\varepsilon}\right|^{2}\right) .
\end{gathered}
$$

Now sending $\varepsilon$ to zero and using (3.8) we obtain that the last inequality is non positive. Thus we obtain a contradiction to (3.11) and $U_{\xi} \leqslant V_{\xi}$ for all $\xi>0$ on $(0, T] \times \mathcal{O}_{+}^{d} \times[0,1]$. This gives the required result by sending $\xi$ to zero.

\section{Application to the quantile hedging of Bermudan op- tions}

In this section we are interested in the case where the loss function is the indicator function leading to a quantile hedging problem. More precisely we are interested in the 
Bermudan version of this problem, i.e. we define for $(t, x, p) \in[0, T] \times \mathcal{O}_{+}^{d} \times[0,1]$

$$
\begin{aligned}
& v(t, x, p):=\inf \left\{y \geqslant 0: \exists \nu \in \mathcal{U}_{t, x, y} \text { s.t. } \mathbb{P}\left[\bigcap_{s \in \mathbb{T}_{t}} \mathrm{~S}_{s}^{t, x, y, \nu}\right] \geqslant p\right\}, \\
& \text { with } \mathrm{S}_{s}^{t, x, y, \nu}:=\left\{\begin{array}{lll}
\Omega & \text { if } & s \leqslant t \\
\left\{Y_{s}^{t, x, y, \nu} \geqslant g\left(s, X_{s}^{t, x}\right)\right\} & \text { if } & s>t
\end{array}\right. \text {, } \\
& \text { and } \mathbb{T}_{t}:=\left\{t_{0}=0 \leqslant \cdots \leqslant t_{i} \leqslant \cdots \leqslant t_{n}=T\right\} \cap(t, T] \text {, }
\end{aligned}
$$

where $g:[0, T] \times \mathcal{O}_{+}^{d} \rightarrow \mathbb{R}^{+}$and $x \in \mathcal{O}_{+}^{d} \mapsto g(t, x)$ is Lipschitz continuous for all $t \in[0, T]$ and where $\mathcal{U}_{t, x, y}$ is defined in Section 2. Observe that $v(t, \cdot)$ must be interpreted as a continuation value, i.e. the price at time $t$ knowing that the option has not been exercised on $[0, t]$. In particular, $v(T, \cdot)=0$ on $\mathcal{O}_{+}^{d} \times[0,1]$.

This problem is equivalent to (see $(2.4)$ )

$v(t, x, p)=\inf \left\{y \in \mathbb{R}^{+}: \exists(\nu, \alpha) \in \mathcal{U}_{t, x, y} \times \mathcal{A}_{t, p}\right.$ s.t. $\left.Y_{s}^{t, x, y, \nu} \geqslant g\left(s, X_{s}^{t, x}\right) \mathbf{1}_{\left\{P_{s}^{t, p, \alpha}>0\right\}}, \forall s \in \mathbb{T}_{t}\right\}$,

where for $p \in[0,1], \mathcal{A}_{t, p}$ is the set of $\mathbb{R}^{d}$-valued $\mathbb{F}$-progressively measurable and square integrable processes $\alpha$ such that $P^{t, p, \alpha} \in[0,1]$ on $[t, T]$.

The aim of this section is to give a characterisation of $v$ as the unique solution to a sequence of PDEs. To this end, and in view of the previous section (recall Remark 3.4), we need in particular the knowledge of $v$ on the boundary $p=0$ and $p=1$. We will show that, as expected, $v(\cdot, 0)=0$ and $v(\cdot, 1)=\bar{v}(\cdot)$, where $\bar{v}$ is the super-replication price of the Bermudan option with exercise price $g$. Moreover, we assume that Assumption 2.0.2 holds for $\bar{v}$ in this Bermudan setting. Therefore, Remark 2.3 is still valid for $v$. Precisely from standard results in stochastic control theory (see [19] for instance) and from [7], we have the following characterisation of $\bar{v}$.

Proposition 4.1. Fix $1 \leqslant i \leqslant n$.

The function $\bar{v}$ is continuous on $\left[t_{i-1}, t_{i}\right) \times \mathcal{O}_{+}^{d}$ and is the unique viscosity solution, in the class of function with polynomial growth, of

$$
\min \left\{\bar{v}(t, x),\left\{\begin{array}{c}
-\partial_{t} \varphi(t, x)+\mu_{Y}\left(t, x, y, \operatorname{diag}[x] \mathrm{D}_{x} \varphi(t, x)\right)-\mu_{X}^{\top}(x) \mathrm{D}_{x} \varphi(t, x) \\
-\frac{1}{2} \operatorname{Tr}\left[\sigma_{X} \sigma_{X}^{\top}(x) \mathrm{D}_{x x} \varphi(t, x)\right]
\end{array}\right\}=0,\right.
$$

with terminal condition at time $t_{i}$,

$$
\lim _{t \uparrow t_{i}} v\left(t_{i}, \cdot\right)=\bar{v}\left(t_{i}, \cdot\right) \vee g\left(t_{i}, \cdot\right)
$$

We can now state the main result of the section which is the full PDE characterisation of $v$, the quantile hedging price of the Bermudan option with exercise price $g$. 
Theorem 4.1. Fix $1 \leqslant i \leqslant n$.

The function $v$ is continuous on $\left[t_{i-1}, t_{i}\right) \times \mathcal{O}_{+}^{d} \times[0,1]$ and is the unique viscosity solution, in the class of functions with polynomial growth, of

$$
\mathcal{H} v(t, x, p)=0, \text { for }(t, x, p) \in\left[t_{i-1}, t_{i}\right) \times \mathcal{O}_{+}^{d} \times(0,1),
$$

with boundary conditions $v(t, x, 0)=0, v(t, x, 1)=\bar{v}(t, x),(t, x) \in\left[t_{i-1}, t_{i}\right) \times \mathcal{O}_{+}^{d}$ and

$$
\lim _{t \uparrow t_{i}} v(t, x, p)=\overline{\operatorname{conv}}\left(v\left(t_{i}, x, p\right) \vee g\left(t_{i}, x\right) \mathbf{1}_{\{p>0\}}\right), \quad(x, p) \in \mathcal{O}_{+}^{d} \times[0,1] .
$$

Using [3, Proposition 3.3 (a)], we observe that the terminal condition at time $t_{i}$, $1 \leqslant i \leqslant n$, can be easily computed. More precisely it is obtained by applying [3, Lemma 3.1(a)], the fact that $v\left(t_{i+1}, \cdot, 0\right)=0$ and the definition of $p_{g}$.

Remark 4.1. (i) The boundary condition at time $t_{i}, 1 \leqslant i \leqslant n$, and for $(x, p) \in$ $\mathcal{O}_{+}^{d} \times[0,1]$, is given by

$$
\overline{\operatorname{conv}}\left(v\left(t_{i}, x, p\right) \vee g\left(t_{i}, x\right) \mathbf{1}_{\{p>0\}}\right)=v\left(t_{i}, x, p\right) \vee \tilde{g}\left(t_{i}, x, p\right),
$$

where for $(t, x, p) \in[0, T] \times \mathcal{O}_{+}^{d} \times \mathbb{R}, \tilde{g}$ is the following 'facelift' of $g$

$$
\tilde{g}(t, x, p)=q_{g}(t, x) p \mathbf{1}_{\{0 \leqslant p \leqslant 1\}}+\infty \mathbf{1}_{\{p>1\}},
$$

with

$$
q_{g}(t, x):=\frac{g(t, x)}{p_{g}(t, x)} \mathbf{1}_{\left\{p_{g}(t, x)>0\right\}} \text { and } p_{g}(t, x):=\sup \{p \in \mathbb{R} \mid v(t, x, p)=g(t, x)\} \wedge 1 .
$$

(ii) In particular, at time $T$, the terminal condition is given by $(t, x, p) \mapsto p g(t, x)$, which was already observed in [7, Proposition 3.2].

\subsection{Proof of Theorem 4.1}

We now turn to the proof of the main result of this section. As usual in the case of Bermudan option, the proof is done by induction on the time interval $\left[t_{i}, t_{i+1}\right)$, $0 \leqslant i \leqslant n-1$. The main difficulty here is the characterisation of $v$ on the boundaries of the domain, specially the time-boundary for which a facelifting phenomenon appears.

The results stated in this section are a direct consequence of the geometric dynamic programming principle, see $[7,20,21]$. In our framework, we obtain from [9, Theorem 2.1 , the following geometric dynamic programming principle, 
(GDP1) Fix $1 \leqslant i \leqslant n$ and $(t, x, p) \in\left[t_{i-1}, t_{i}\right) \times \mathcal{O}_{+}^{d} \times[0,1]$. If $y>v(t, x, p)$, then there exists a $(\nu, \alpha) \in \mathcal{U}_{t, x, y} \times \mathcal{A}_{t, p}$ such that for all stopping times $\theta \leqslant t_{i}$

$$
Y_{\theta}^{t, x, y, \nu} \geqslant v\left(\theta, X_{\theta}^{t, x}, P_{\theta}^{t, p, \alpha}\right) \mathbf{1}_{\left\{\theta<t_{i}\right\}}+(v \vee g)\left(t_{i}, X_{t_{i}}^{t, x}, P_{t_{i}}^{t, p, \alpha}\right) \mathbf{1}_{\left\{\theta=t_{i}\right\}} .
$$

(GDP2) Fix $1 \leqslant i \leqslant n$ and $(t, x, p) \in\left[t_{i-1}, t_{i}\right) \times \mathcal{O}_{+}^{d} \times[0,1]$. For every $y<v(t, x, p)$, $(\nu, \alpha) \in \mathcal{U}_{t, x, y} \times \mathcal{A}_{t, p}$ and all stopping times $\theta \leqslant t_{i}$

$$
\mathbb{P}\left[Y_{\theta}^{t, x, y, \nu}>v\left(\theta, X_{\theta}^{t, x}, P_{\theta}^{t, p, \alpha}\right) \mathbf{1}_{\left\{\theta<t_{i}\right\}}+(v \vee g)\left(t_{i}, X_{t_{i}}^{t, x}, P_{t_{i}}^{t, p, \alpha}\right) \mathbf{1}_{\left\{\theta=t_{i}\right\}}\right]<1,
$$

with the notation $g\left(t_{i}, x, p\right):=g\left(t_{i}, x\right) \mathbf{1}_{p>0}+\infty \mathbf{1}_{p>1}, 1 \leqslant i \leqslant n$ and $x \in \mathcal{O}_{+}^{d}$.

Let us now proceed with the proof of Theorem 4.1.

For $i \leqslant n-1$, we now assume that $v\left(t_{i+1}, \cdot\right)$ is continuous, $v\left(t_{i+1}, \cdot, 0\right)=0$ and $v\left(t_{i+1}, \cdot, 1\right)=\bar{v}\left(t_{i+1}, \cdot\right)$. (Observe that this is the case by convention at time $T$ as $v(T, \cdot)=0=\bar{v}(T, \cdot))$.

To clarify the arguments, we introduce the following function on $\left[t_{i}, t_{i+1}\right] \times \mathcal{O}_{+}^{d} \times[0,1]$

$$
\hat{v}(t, x, p):=\left\{\begin{array}{l}
v(t, x, p) \text { on }\left[t_{i}, t_{i+1}\right) \times \mathcal{O}_{+}^{d} \times[0,1] \\
v\left(t_{i+1}, x, p\right) \vee g\left(t_{i+1}, x\right) \mathbf{1}_{p>0} \text { on } \mathcal{O}_{+}^{d} \times[0,1]
\end{array} .\right.
$$

Step 1 Characterisation on $\left[t_{i}, t_{i+1}\right) \times \mathcal{O}_{+}^{d} \times[0,1]$.

From (GDP1) - (GDP2), combining the results of [7, Theorem 2.1] and Theorem 3.1, we obtain that $\hat{v}$ is a viscosity solution of

$$
\mathcal{H} \hat{v}=0, \text { on }\left[t_{i}, t_{i+1}\right) \times \mathcal{O}_{+}^{d} \times(0,1) .
$$

Moreover applying [7, Theorem 3.1] we obtain that, on $\left[t_{i}, t_{i+1}\right) \times \mathcal{O}_{+}^{d}$,

$$
\hat{v}^{*}(\cdot, 1)=\hat{v}_{*}(\cdot, 1)=\bar{v}(\cdot) \text { and } \hat{v}^{*}(\cdot, 0)=\hat{v}_{*}(\cdot, 0)=0 .
$$

Step 2 Characterisation on $\left\{t_{i+1}\right\} \times \mathcal{O}_{+}^{d} \times[0,1]$.

Step 2.a We first prove that

$$
\hat{v}^{*}\left(t_{i+1}, x, p\right) \leqslant \overline{\mathrm{conv}}\left(v\left(t_{i+1}, x, p\right) \vee g\left(t_{i+1}, x\right) \mathbf{1}_{\{p>0\}}\right) .
$$

Proceeding as in $[7$, Section 5.4] we first obtain

$$
\hat{v}^{*}\left(t_{i+1}, x, p\right) \leqslant\left(v\left(t_{i+1}, x, p\right) \vee g\left(t_{i+1}, x\right) \mathbf{1}_{\{p>0\}}\right)^{*} .
$$

Now, it follows from the sub-solution property that, for any test function $\varphi$ such that $\max _{\left[t_{i}, t_{i+1}\right) \times \mathcal{O}_{+}^{d} \times(0,1)}\left(\hat{v}^{*}-\varphi\right)(t, x, p)=0$, we have $\mathrm{D}_{p p} \varphi(t, x, p) \geqslant 0$ (recall Remark 3.2 
(1)). Applying then the same argument as in [13, Proposition 5.2] we conclude that $\hat{v}^{*}$ is convex inside the domain. From the upper semi-continuity of $\hat{v}^{*}$, we obtain the convexity property in the $p$-variable on $[0,1]$.

Combining (4.2) and (4.3), we observe that

$$
\hat{v}^{*}\left(t_{i+1}, x, p\right) \leqslant v\left(t_{i+1}, x, p\right) \vee g\left(t_{i+1}, x\right) \mathbf{1}_{p>0} .
$$

We now use (4.1) and (4.4). Indeed, we observe that, for all $p \in\left[0, p_{g}\left(t_{i+1}, x\right)\right]$,

$$
\hat{v}^{*}\left(t_{i+1}, x, p\right) \leqslant v\left(t_{i+1}, x, p\right) \vee \tilde{g}\left(t_{i+1}, x, p\right),
$$

since $p \mapsto \hat{v}^{*}(\cdot, p)$ is convex, $\hat{v}^{*}\left(t_{i+1}, x, 0\right)=0$ and

$$
\hat{v}^{*}\left(t_{i+1}, x, p_{g}\left(t_{i+1}, x\right)\right) \leqslant v\left(t_{i+1}, x, p_{g}\left(t_{i+1}, x\right)\right)=g\left(t_{i+1}, x\right) .
$$

For $p \in\left[p_{g}\left(t_{i+1}, x\right), 1\right]$, we have that

$$
\hat{v}^{*}\left(t_{i+1}, x, p\right) \leqslant v\left(t_{i+1}, x, p\right) \vee g\left(t_{i+1}, x\right)=v\left(t_{i+1}, x, p\right)=v\left(t_{i+1}, x, p\right) \vee \tilde{g}\left(t_{i+1}, x, p\right),
$$

which concludes the proof for this step.

Step 2.b We now prove

$$
\hat{v}_{*}\left(t_{i+1}, x, p\right) \geqslant \overline{\operatorname{conv}}\left(v\left(t_{i+1}, x, p\right) \vee g\left(t_{i+1}, x\right) \mathbf{1}_{\{p>0\}}\right) .
$$

To obtain the above result, we will use the following Lemma, whose proof is postponed at the end of this section.

Lemma 4.1. For all sequences $\left(t_{k}, x_{k}, p_{k}, y_{k}, \nu^{k}, \alpha^{k}\right)_{k \geqslant 1} \in\left[t_{i}, t_{i+1}\right) \times \mathcal{O}_{+}^{d} \times(0,1) \times \mathbb{R}^{+} \times$ $\mathcal{U}_{t_{k}, x_{k}, y_{k}} \times \mathcal{A}_{t_{k}, p_{k}}, 0 \leqslant i \leqslant n-1$ such that $\left(t_{k}, x_{k}, p_{k}, y_{k}\right) \rightarrow\left(t_{i+1}, x, p, y\right) \in \mathcal{O}_{+}^{d} \times[0,1] \times$ $\mathbb{R}^{+}$, there exists a sequence of non-negative random variables $\left(H_{t_{i+1}}^{k}\right)_{k \geqslant 1}$, such that

$$
\limsup _{k \rightarrow \infty} \mathbb{E}\left[H_{t_{i+1}}^{k} Y_{t_{i+1}}^{t_{k}, x_{k}, y_{k}, \nu^{k}}\right] \leqslant y \quad \text { and } \quad \liminf _{k \rightarrow \infty} H_{t_{i+1}}^{k}=1 \text {. }
$$

Fix $(x, p) \in \mathcal{O}_{+}^{d} \times[0,1]$. Let $\left(t_{k}, x_{k}, p_{k}\right)_{k \geqslant 1} \in\left[t_{i}, t_{i+1}\right) \times \mathcal{O}_{+}^{d} \times(0,1)$ be a sequence such that $\left(t_{k}, x_{k}, p_{k}\right) \rightarrow\left(t_{i+1}, x, p\right)$ and $\hat{v}\left(t_{k}, x_{k}, p_{k}\right) \rightarrow \hat{v}_{*}\left(t_{i+1}, x, p\right)$. Set for every $k \geqslant 1$, $y_{k}:=\hat{v}\left(t_{k}, x_{k}, p_{k}\right)+k^{-1}$ so that by (GDP1) there exists $\left(\nu^{k}, \alpha^{k}\right) \in \mathcal{U}_{t_{k}, x_{k}, y_{k}} \times \mathcal{A}_{t_{k}, p_{k}}$ such that

$$
Y_{t_{i+1}}^{t_{k}, x_{k}, y_{k}, \nu^{k}} \geqslant v\left(t_{i+1}, X_{t_{i+1}}^{t_{k}, x_{k}}, P_{t_{i+1}}^{t_{k}, p_{k}, \alpha^{k}}\right) \vee g\left(t_{i+1}, X_{t_{i+1}}^{t_{k}, x_{k}}\right) \mathbf{1}_{\left\{P_{t_{i+1}}^{t_{k}, p_{k}, \alpha^{k}}>0\right\}} .
$$


Now first multiply by $H_{t_{i+1}}^{k}$ and then take the expectation and the limit to obtain with Fatou's Lemma

$$
\begin{aligned}
\liminf _{k \rightarrow \infty} & \mathbb{E}\left[H_{t_{i+1}}^{k} Y_{t_{i+1}}^{t_{k}, x_{k}, y_{k}, \nu^{k}}\right] \\
& \geqslant \mathbb{E}\left[\liminf _{k \rightarrow \infty}\left(H_{t_{i+1}}^{k}\left(v\left(t_{i+1}, X_{t_{i+1}}^{t_{k}, x_{k}}, P_{t_{i+1}}^{t_{k}, p_{k}, \alpha^{k}}\right) \vee g\left(t_{i+1}, X_{t_{i+1}}^{t_{k}, x_{k}}\right) \mathbf{1}_{\left\{P_{t_{i+1}}^{t_{k}, p_{k}, \alpha^{k}}>0\right\}}\right)\right)\right] .
\end{aligned}
$$

We then use the Lipschitz continuity of $g$, the continuity of $v$, the lower semi-continuity of $r \in[0,1] \mapsto \mathbf{1}_{r>0}$, the $L^{1}$ convergence of $\left(X_{t_{i+1}}^{t_{k}, x_{k}}, P_{t_{i+1}}^{t_{k}, p_{k}, \alpha^{k}}\right)$ towards $(x, p)$ and (4.5) to obtain

$$
\begin{aligned}
\hat{v}_{*}\left(t_{i+1}, x, p\right) \geqslant \liminf _{k \rightarrow \infty} \mathbb{E}\left[H_{t_{i+1}}^{k} Y_{t_{i+1}}^{t_{k}, x_{k}, y_{k}, \nu^{k}}\right] & \geqslant v\left(t_{i+1}, x, p\right) \vee g\left(t_{i+1}, x\right) \mathbf{1}_{\{p>0\}} \\
& \geqslant \overline{\operatorname{conv}}\left(v\left(t_{i+1}, x, p\right) \vee g\left(t_{i+1}, x\right) \mathbf{1}_{\{p>0\}}\right),
\end{aligned}
$$

by definition of the closed convex envelope.

Step 3 To conclude, let us observe that by using the $p$-boundary condition on $\hat{v}^{*}$ and $\hat{v}_{*}$ in equation (4.2), the time-boundary condition of the previous step and the comparison theorem proved in the last section, we obtain that $\hat{v}$ is continuous on $\left[t_{i}, t_{i+1}\right) \times \mathcal{O}_{+}^{d} \times$ $[0,1]$.

Proof of Lemma 4.1. For ease of notations, we introduce $Y^{k}:=Y^{t_{k}, x_{k}, y_{k}, \nu^{k}}$, $X^{k}:=X^{t_{k}, x_{k}}$. For later use, let us also observe that under the standing assumptions on the coefficients of $X^{k}$, the following holds true

$$
\mathbb{E}\left[\left|X_{t}^{k}\right|^{q}\right] \leqslant C_{q}, \text { for all } q \geqslant 1,
$$

where $C_{q}>0$ is a constant that does not depend on $k$. We now define $\tilde{Y}^{k}:=$ $e^{-L\left(t-t_{k}\right)} H_{t}^{k} Y_{t}^{k}, t \in\left[t_{k}, t_{i+1}\right]$ where $H^{k}$ is the solution to

$$
H_{t}^{k}=1-\int_{t_{k}}^{t} L H_{s}^{k}\left\{\sigma^{-1}\left(X_{s}^{k}\right) \beta_{s}^{k}\right\}^{\top} \mathrm{d} W_{s}, \quad \text { for } t \in\left[t_{k}, t_{i+1}\right],
$$

with $\beta^{k}=\left(\operatorname{sign}\left[\left(\nu^{k}\right)^{i}\right]\right)_{1 \leqslant i \leqslant d}$ and $L$ defined in (2.1). As $\sigma^{-1}$ is bounded and $\left|\beta^{k}\right| \leqslant d$, we have that

$$
\mathbb{E}\left[\left(H_{t}^{k}\right)^{q}\right] \leqslant C_{q}, \text { for all } q \geqslant 1,
$$

where $C_{q}>0$ is a constant that does not depend on $k$. In particular, we observe that $\liminf \inf _{k \rightarrow \infty} H_{t_{i+1}}^{k}=1$.

Now, applying Ito's formula, we compute

$\tilde{Y}_{t}^{k}=y+\int_{t_{k}}^{t}\left(e^{-L\left(s-t_{k}\right)} H_{s}^{k} \mu_{Y}\left(s, X_{s}^{k}, Y_{s}^{k}, \nu_{s}^{k}\right)-L \tilde{Y}_{s}-L e^{-L\left(s-t_{k}\right)} H_{s}^{k}\left(\nu_{s}^{k}\right)^{\top} \beta_{s}^{k}\right) \mathrm{d} s+M_{t}^{k}-M_{t_{k}}^{k}$, 
for some local martingale $M^{k}$. It is easily seen that this local martingale is actually a super-martingale as it is bounded from below by an integrable term. Using (2.1), observing that $\left(\nu_{s}^{k}\right)^{\top} \beta_{s}^{k}=\left|\nu_{s}^{k}\right|$ and recalling $Y \geqslant 0$, we obtain

$$
0 \leqslant \mathbb{E}\left[\tilde{Y}_{t}^{k}\right] \leqslant y+C \int_{t_{k}}^{t}\left(1+\mathbb{E}\left[\left(1+\left|\nu_{s}^{k}\right|\right) H_{s}^{k}\left|X_{s}^{k}\right|\right]\right) \mathrm{d} s .
$$

Using Cauchy-Schwarz's inequality, (4.6)-(4.7) and the square integrability of $\nu_{s}^{k}$, we get, recalling the definition of $\tilde{Y}^{k}$,

$$
\mathbb{E}\left[H_{t}^{k} Y_{t}^{k}\right] \leqslant e^{L\left(t-t_{k}\right)}\left(y+C\left(t-t_{k}\right)\right), \text { for all } t \in\left[t_{k}, t_{i+1}\right],
$$

which concludes the proof of the lemma.

\section{References}

[1] Olivier Bokanowski, Benjamin Bruder, Stefania Maroso, and Hasnaa Zidani. Numerical approximation for a superreplication problem under gamma constraints. SIAM Journal on Numerical Analysis, 47(3):2289-2320, 2009.

[2] Olivier Bokanowski, Athena Picarelli, and Hasnaa Zidani. State-constrained stochastic optimal control problems via reachability approach. SIAM Journal on Control and Optimization, 54(5):2568-2593, 2016.

[3] Bruno Bouchard, Géraldine Bouveret, and Jean-François Chassagneux. A backward dual representation for the quantile hedging of Bermudan options. SIAM Journal on Financial Mathematics, 7(1):215-235, 2016.

[4] Bruno Bouchard and Ngoc-Minh Dang. Generalized stochastic target problems for pricing and partial hedging under loss constraints - application in optimal book liquidation. Finance and Stochastics, 17(1):31-72, 2013.

[5] Bruno Bouchard, Romuald Elie, and Antony Réveillac. BSDEs with weak terminal condition. preprint, 2012. Cited on, pages 2,7.

[6] Bruno Bouchard, Romuald Elie, and Antony Réveillac. BSDEs with weak terminal condition. The Annals of Probability, 43(2):572-604, 2015.

[7] Bruno Bouchard, Romuald Elie, and Nizar Touzi. Stochastic target problems with controlled loss. SIAM Journal on Control and Optimization, 48(5):3123-3150, 2009 . 
[8] Bruno Bouchard and Thanh Nam Vu. A stochastic target approach for P\&L matching problems. Mathematics of Operations Research, 37(3):526-558, 2012.

[9] Bruno Bouchard and Thanh Nam Vu. The obstacle version of the geometric dynamic programming principle: Application to the pricing of American options under constraints. Applied Mathematics and Optimization, 61(2):235-265, 2010.

[10] Benjamin Bruder. Super-replication of European options with a derivative asset under constrained finite variation strategies. preprint, 2005.

[11] Patrick Cheridito, H Mete Soner, and Nizar Touzi. The multi-dimensional superreplication problem under gamma constraints. 22(5):633-666, 2005.

[12] Michael G Crandall, Hitoshi Ishii, and Pierre-Louis Lions. Users guide to viscosity solutions of second order partial differential equations. Bulletin of the American Mathematical Society, 27(1):1-67, 1992.

[13] Jakša Cvitanić, Huyen Pham, and Nizar Touzi. A closed-form solution to the problem of super-replication under transaction costs. Finance and stochastics, 3(1):35-54, 1999.

[14] Nicole El Karoui, Shige Peng, and Marie Claire Quenez. Backward stochastic differential equations in finance. Mathematical finance, 7(1):1-71, 1997.

[15] Hans Föllmer and Peter Leukert. Quantile hedging. Finance and Stochastics, 3(3):251-273, 1999.

[16] Hitoshi Ishii and Pierre-Louis Lions. Viscosity solutions of fully nonlinear secondorder elliptic partial differential equations. Journal of Differential equations, 83(1):26-78, 1990.

[17] Ying Jiao, Olivier Klopfenstein, and Peter Tankov. Hedging under multiple risk constraints. arXiv preprint arXiv:1309.5094, 2013.

[18] Ludovic Moreau. Stochastic target problems with controlled loss in jump diffusion models. SIAM Journal on Control and Optimization, 49(6):2577-2607, 2011.

[19] Huyên Pham. Continuous-time stochastic control and optimization with financial applications, volume 61. Springer Science \& Business Media, 2009.

[20] H Mete Soner and Nizar Touzi. Dynamic programming for stochastic target problems and geometric flows. Journal of the European Mathematical Society, 4(3):201236, 2002. 
[21] H Mete Soner and Nizar Touzi. Stochastic target problems, dynamic programming, and viscosity solutions. SIAM Journal on Control and Optimization, 41(2):404424, 2002. 